\title{
Magneto-Optic Tracking of a Flexible Laparoscopic Ultrasound Transducer for Laparoscope Augmentation
}

\author{
Marco Feuerstein ${ }^{1}$, Tobias Reichl ${ }^{1}$, Jakob Vogel ${ }^{1}$, Armin Schneider ${ }^{2}$, \\ Hubertus Feussner ${ }^{2}$, and Nassir Navab ${ }^{1}$ \\ ${ }^{1}$ Computer-Aided Medical Procedures (CAMP), TUM, Munich, Germany \\ ${ }^{2}$ Department of Surgery, Klinikum rechts der Isar, TUM, Munich, Germany
}

\begin{abstract}
In abdominal surgery, a laparoscopic ultrasound transducer is commonly used to detect lesions such as metastases. The determination and visualization of position and orientation of its flexible tip in relation to the patient or other surgical instruments can be of much help to (novice) surgeons utilizing the transducer intraoperatively. This difficult subject has recently been paid attention to by the scientific community

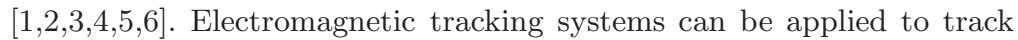
the flexible tip. However, the magnetic field can be distorted by ferromagnetic material. This paper presents a new method based on optical tracking of the laparoscope and magneto-optic tracking of the transducer, which is able to automatically detect field distortions. This is used for a smooth augmentation of the B-scan images of the transducer directly on the camera images in real time.
\end{abstract}

\section{Introduction}

Laparoscopic ultrasonography (LUS) nowadays plays an increasing role in abdominal surgery. Its main application areas include liver, biliary tract, and pancreas. Unfortunately LUS is often difficult to perform, especially for novice surgeons. Therefore, several groups tried to support surgeons by providing navigated LUS: The position and orientation ("pose") of the ultrasound transducer is estimated, so its body and B-scan images can be visualized in relation to the patient, other surgical instruments, or preoperative and intraoperative imaging data. This may greatly support surgeons utilizing LUS in cancer staging, radio frequency ablation, and other procedures.

To estimate the pose of a transducer with a rigid tip, a robot or optical tracking (OT) may be used [2]. In the latter case, a rigid body can be attached to the transducer handle to assure its continuous visibility. Several groups also try to localize rigid laparoscopic instruments in laparoscopic images by advanced image processing techniques, such as Voros et al. [7. However, laparoscopic transducers most commonly used and preferred by surgeons feature a flexible tip providing rightward, leftward, forward, and backward steering. The tip also yields to external pressure from organ surfaces. Due to the missing line of sight to the flexible 
transducer tip, an OT system cannot be used exclusively to localize this tip. A robot could only be utilized if the ultrasound probe was fully integrated into the end-effector. To the authors' knowledge no such system currently exists. Promising alternatives are the use of an electromagnetic tracking (EMT) sensor attached to the tip 456] or fully incorporated into the tip [1, or magneto-optic tracking, i.e. the combination of OT and EMT [3].

When clinically using EMT, a considerable problem is the distortion of the EMT field leading to erroneous tracking data. This distortion can be caused by metallic or electrically powered objects inside or in close vicinity to the working volume, for instance surgical instruments, an operating table, or imaging devices such as a C-arm or a computed tomography scanner. Depending on the operating room setup and instrumentation, tracking errors of several millimeters or even centimeters can occur [8]. To compensate for erroneous measurements caused by stationary objects, various calibration techniques were proposed [10]. They usually require the user to acquire a set of well distributed measurements within the EMT volume. This set is compared to a set of reference measurements to compute a field distortion function that is based on look-up tables or polynomials. Unfortunately, this function can only compensate static errors of non-moving distortion fields, so that the calibration process has to be repeated for every new operating room setup before an intervention. Dynamic changes of the field distortion, for example caused by the intraoperative relocation of the EMT transmitter or movement of instruments, cannot be compensated by the previously computed distortion functions. A first step towards the intraoperative detection of erroneous measurements caused by metallic objects distorting the field was presented by Birkfellner et al. [11. They incorporate two sensors into a pointer, so redundant measurements can be obtained. Deviations of the fixed distance between the two sensors are used as a plausibility value.

This paper introduces a new method to detect field distortions online, i.e. intraoperatively without a pre-computed distortion function. It is applied to a flexible laparoscopic transducer whose pose is determined by a magneto-optic tracking system. The B-scan images of the transducer are overlaid on the live images of an optically tracked laparoscope in real time to provide surgeons with a better understanding of the spatial relationship between the two imaging modalities. Finally, a rigorous accuracy evaluation of both online field distortion estimation and laparoscope augmentation is presented.

\section{System Setup}

The hardware setup comprises following components: A flexible laparoscopic linear array transducer (LAP8-4, $5 \mathrm{MHz}, 10 \mathrm{~mm}$ diameter) connected to a SONOLINE Omnia US system by Siemens Medical Solutions, a laparoscopic camera with a forward-oblique $30^{\circ}$ HOPKINS telescope by Storz, a standard workstation PC including two frame grabbers (for capturing the transducer and camera video in real time), and the magneto-optic tracking system. The OT system consists of 4 ARTtrack2 cameras and a book size PC running the DTrack tracking 
software. The EMT system in use is a 3D Guidance unit of Ascension equipped with a mid-range transmitter and insulated $1.3 \mathrm{~mm}$ sensors, which have a total diameter of $1.7 \mathrm{~mm}$ including the vinyl tubing. Time synchronization of all data streams and visualization is performed by CAMPAR 12 .

\section{Methods}

In addition to an OT body, which is attached to the transducer handle (below referred to as "rigid body"), two EMT sensors are attached to the transducer shaft: One to the flexible tip ("flexible sensor"), the other one to the rigid part ("rigid sensor"), as close to each other as possible. Another OT body is mounted on the EMT transmitter ("transmitter body"). This setup allows us to co-calibrate EMT and OT and to obtain redundant tracking information of the rigid part of the transducer shaft, which is important to detect EMT errors. Finally, two OT bodies are attached to the laparoscopic camera, one to the head ("laparoscope body") and another one to the telescope to adjust for telescope rotations.

\subsection{System Calibration}

Spatial and temporal system calibration is performed offline in a distortion-free environment. All coordinate frames are visualized in figure 1.

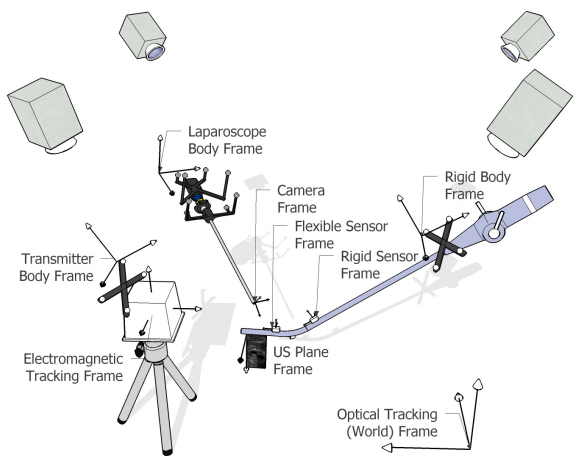

Fig. 1. Coordinate frames

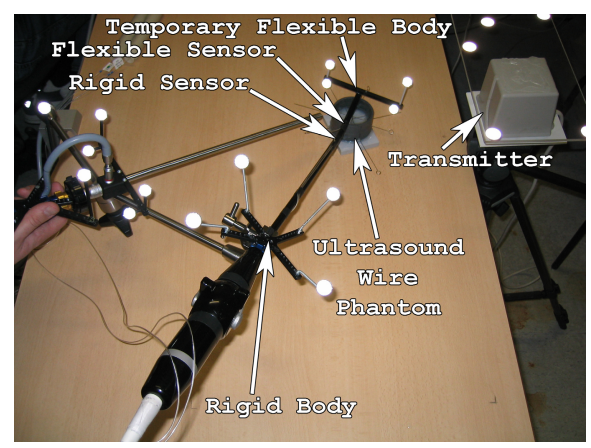

Fig. 2. Setup for error evaluation

Hand-eye Calibration. To compute the Euclidean transformation ${ }^{R i g B} \boldsymbol{T}_{\text {RigS }}$ between the rigid body and the rigid sensor frames, several poses with distinct rotation axes are recorded in both the OT and EMT coordinate frames. Stacked matrices $\boldsymbol{A}$ and $\boldsymbol{B}$ are generated from all movements between these poses. They are related to each other by the equation system $\boldsymbol{A} \boldsymbol{X}=\boldsymbol{X} \boldsymbol{B}$, which is solved by hand-eye calibration [13. The same poses are used to estimate the rigid handeye transformation ${ }^{E M T} \boldsymbol{T}_{\text {TransB }}$ between the EMT transmitter coordinate frame and its OT body. 
In a final optimization step, the two hand-eye calibration matrices ${ }^{R i g B} \boldsymbol{T}_{\text {RigS }}$ and ${ }^{E M T} \boldsymbol{T}_{\text {TransB }}$ are optimized for all recorded poses by Levenberg-Marquardt. The matrix resulting from the transformation chain "rigid sensor to rigid body to OT to transmitter body to EMT to rigid sensor frame", which theoretically is an identity matrix, represents the accumulated transformation errors:

$$
\boldsymbol{T}_{\delta}=\left[\begin{array}{cc}
\boldsymbol{R}_{\delta} & \boldsymbol{t}_{\delta} \\
0 & 1
\end{array}\right]={ }^{R i g S} \boldsymbol{T}_{E M T} \cdot{ }^{E M T} \boldsymbol{T}_{\text {Trans } B} \cdot{ }^{\operatorname{Trans} B} \boldsymbol{T}_{O T}{ }^{O T} \boldsymbol{T}_{\text {RigB }} \cdot{ }^{\text {RigB }} \boldsymbol{T}_{R i g S}
$$

We chose a cost function $\delta$ that weights translational to rotational errors 1:3, reflecting the root mean squared (RMS) error ratio provided independently by the two tracking system manufacturers: The RMS measurement errors of the OT system are stated as $0.4 \mathrm{~mm}$ (position) and $0.12^{\circ}$ (orientation), the static RMS errors of the EMT system as $1.4 \mathrm{~mm}$ and $0.5^{\circ} 1$

$$
\delta=\delta_{\text {translational }}+3 \cdot \delta_{\text {rotational }}=\left\|\boldsymbol{t}_{\delta}\right\|+3 \cdot \frac{180}{\pi} \cdot \arccos \left(\frac{\operatorname{trace}\left(\boldsymbol{R}_{\delta}\right)-1}{2}\right)
$$

where the rotational error is the rotation angle of $\boldsymbol{R}_{\delta}$, decomposed into axis-angle parameters. The maximum error $\delta_{\max }$ determined after optimization is chosen as a measure of distrust for the overall performance of the hand-eye calibration (cf. section 3.2).

Laparoscopic Camera. For laparoscopic camera calibration, the projection geometry including distortion coefficients and the transformation from laparoscope body coordinates to camera center coordinates are estimated, as described by Yamaguchi et al. [14.

Laparoscopic Ultrasound. For the determination of the pixel scaling of the ultrasound B-scan plane and its transformation to the flexible sensor frame, a single-wall calibration is performed [15. Instead of scanning the planar bottom of a water bath, we scan a nylon membrane stretched over a planar frame, as proposed by Lang $\varnothing$ [16].

Temporal Calibration. In order to provide a smooth visualization without lag, all data is given a time stamp and brought into the same time frame. While the OT PC and our workstation are synchronized via the network time protocol (NTP) to the same reference time, the ultrasound and EMT systems require a more advanced synchronization. As these systems do not automatically provide reliable time stamps corresponding to the actual data acquisition time, a time stamp is generated when their data arrives at the workstation. Therefore, a fixed offset is subtracted from this time stamp to compensate for any lag introduced while traveling to the workstation. To determine this offset, the magneto-optically tracked transducer is moved up and down and the translation along the principal motion axes is compared, as proposed by Treece et al. [15].

${ }^{1}$ See also http://www . ar-tracking.de and http://www . ascension-tech.com 


\subsection{Online Error Estimation}

Intraoperatively, every measured pose of the rigid sensor is transformed applying equation 1. If a corresponding error $\delta$ is determined, which is bigger than the distrust level $\delta_{\max }$, the surgical staff is automatically warned. Such errors are often caused by dynamic or static field distortions. Additionally, as the flexible sensor is in close proximity to the rigid one, its measurements will be most likely affected by these distortions as well.

In order to also approximate a correction of erroneous measurements of the flexible sensor, a simple approach is to apply the deviation between the previously hand-eye calibrated ("calib") and the measured ("meas") transformation of the rigid sensor to the measured flexible sensor transformation, all relatively to the fixed OT (world) reference frame:

$$
\begin{aligned}
{ }^{O T} \boldsymbol{R}_{\text {Flex } S(\text { corr })} & ={ }^{O T} \boldsymbol{R}_{\text {Rigid } S(\text { meas })}{ }^{T} \cdot{ }^{O T} \boldsymbol{R}_{\text {Rigid } S(\text { calib })} \cdot{ }^{O T} \boldsymbol{R}_{\text {FlexS(meas })} \\
{ }^{O T} \boldsymbol{t}_{\text {Flex } S(\text { corr })} & =-{ }^{O T} \boldsymbol{t}_{\text {RigidS(meas })}+{ }^{O T} \boldsymbol{t}_{\text {Rigid } S(\text { calib })}+{ }^{O T} \boldsymbol{t}_{\text {FlexS }(\text { meas })}
\end{aligned}
$$

\section{Experimental Evaluation Results}

To avoid too many outliers, all EMT measurements were acquired in a restricted volume of $20-36 \mathrm{~cm}$ for $\mathrm{x}$, and $\pm 15 \mathrm{~cm}$ for $\mathrm{y}$ and $\mathrm{z}$.

\subsection{Ultrasound Calibration Error}

After acquiring 40 flexible sensor poses and their corresponding lines that were automatically detected in the B-scan images, the calibration matrix was computed using the Levenberg-Marquardt optimizer. To determine the ultrasound calibration accuracy, a single EMT sensor with tip coordinates given in the EMT frame was submerged into the water bath. Its tip was segmented manually in 5 regions of the B-scan plane, which was repeated for 4 poses of the transducer differing from the ones used during calibration. The tip coordinates were transformed into the B-scan plane coordinates and compared to the segmented tip coordinates (scaled to $\mathrm{mm}$ ). An RMS error of $1.69 \mathrm{~mm}$ with standard deviation of $0.51 \mathrm{~mm}$ and maximum error of $2.39 \mathrm{~mm}$ was obtained.

\subsection{Laparoscope Augmentation Error}

In order to estimate the laparoscope augmentation errors automatically, an additional OT body ("flexible body") was temporarily attached to the transducer tip and co-calibrated to the flexible sensor by another hand-eye calibration (cf. section 3.1 and figure 2). One marker of the flexible body was chosen as a reference and automatically segmented whenever visible in the laparoscopic video. We compared its center coordinates to the projection of its respective OT coordinates onto the image plane. Additionally, the corresponding EMT measurements as well as their approximated corrections were projected using the previously determined hand-eye calibration transformations. 
Evaluation data was recorded using a laparoscope-to-marker distance of 5 to $10 \mathrm{~cm}$, which is a typical intraoperative working distance. The current distance can be recovered from $\mathrm{OT}$ data and the camera calibration parameters. We also used this information to scale pixel units to mm.

For each of six evaluation series, the transducer was fixed at a different

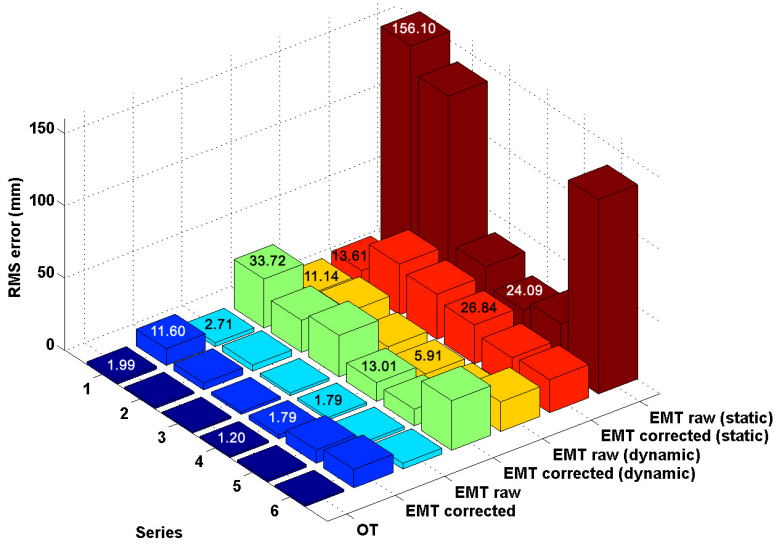

Fig. 3. RMS projection errors pose and the laparoscope was used to measure the projected distances from five differing poses, each in an undistorted and a distorted environment. To distort the EMT field, two alternatives were evaluated. A metal plate was placed on the table to simulate primarily static distortions caused for instance by an operating table. For dynamic distortions, a steel rod of $10 \mathrm{~mm}$ diameter was brought close to the transducer to simulate a surgical instrument, changing its proximity and angle to the transducer in five measurements.

The RMS errors are given in figure 3. For each of the six series, we plotted the errors of the three distortion cases (no distortion, static, and dynamic distortion), each scenario with our simple correction function enabled and disabled. While we have been able to predict and correct static interferences with high reliability, dynamic distortions yielded even worse results when attempting a correction.

In order to evaluate our distrust function statistically, we computed the distrust level (cf. equation 2) for each of the poses. An offset between the segmented marker and the EMT projections of more than $2 \mathrm{~mm}$ was regarded as erroneous measurement. In this case, we expect a distrust level $\delta$ of more than $\delta_{\max }$ (during hand-eye calibration, $\delta_{\max }$ was empirically determined to be 20 ). We defined the following cases for our evaluation:

- A true positive is a measurement, in which the EMT error was above $2 \mathrm{~mm}$ with a distrust level of above 20 - the detector rejected an erroneous reading correctly.

- A true negative is a measurement, in which the EMT error was below $2 \mathrm{~mm}$ with a distrust level below 20 - we correctly accepted the original EMT data.

- A false positive (type 1 error) is a measurement, in which the EMT error was below $2 \mathrm{~mm}$, but the distrust level above 20 - we have not been able to detect a correct value and rejected it without necessity. 
Table 1. Distortion detection rate by our distrust level

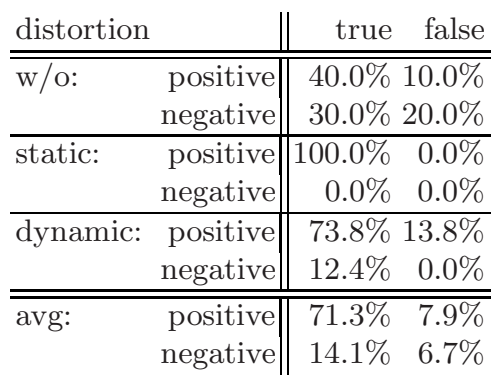

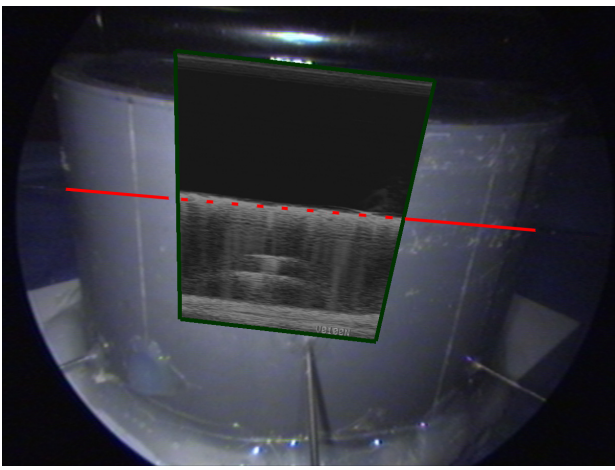

Fig. 4. Ultrasound plane augmented on the laparoscope video - Red line added manually to visualize the extension of the straight wire, which matches its ultrasound image

- A false negative (type 2 error) is a measurement, in which the EMT error was above $2 \mathrm{~mm}$, but the distrust level below 20 - the record was accepted although the real error was large.

The results are listed in table 1 In about $85 \%$ of all cases, we correctly detected the true situation (true positives and true negatives).

\subsection{Ultrasound Augmentation}

To visually inspect the overlay of the B-scan plane on the laparoscopic live video, we constructed a cylindric phantom containing straight wires, which extend through the walls of the phantom. It was filled with water of known temperature. Adjusting the pixel scaling factors to an adequate speed of sound, the B-scan plane was augmented, allowing the camera to view a wire on the augmented plane and its extension outside the phantom walls. A typical augmented laparoscope image can be seen in figure 4

Whenever the occurrence of an error is determined, it is visualized by drawing a red frame around the ultrasound plane. Otherwise the frame is drawn in green. An attempt to correct the error can be visualized in yellow. The supplementary video demonstration 2 summarizes the results of all experiments and allows the observer to qualitatively evaluate the performance of automatic distortion estimation.

\section{Discussion}

The flat tablet transmitter recently presented by Ascension may be an alternative to overcome field distortions, e.g. caused by the operating table. However,

2 http://campar.in.tum.de/files/publications/feuerste2007miccai.video.avi 
due to its lower excitation, in the same setup it performed worse than the midrange transmitter for ultrasound calibration, resulting in errors of about 4-8 mm. Bigger sensors could be used to improve the accuracy, but this would probably require bigger trocars. Using $1.3 \mathrm{~mm}$ sensors, the total diameter of the laparoscopic transducer is only $11.8 \mathrm{~mm}$ (including sterile cover), so it still fits a regular $12 \mathrm{~mm}$ trocar.

In gastrointestinal (laparoscopic) surgery conditions are different than in e.g. orthopedic surgery or neurosurgery. A discrimination of about $0.5 \mathrm{~cm}$ is usually sufficient for a number of reasons. Canalicular structures such as vessels, bile ducts, etc. play a critical role if they are equal to or thicker than $5 \mathrm{~mm}$. Lymph nodes are considered to be inflicted by a tumor if the diameter is more than $10 \mathrm{~mm}$ and so on. Accordingly, an error of about 2-3 $\mathrm{mm}$ (as obtained for the distortion free environment) is certainly acceptable under clinical conditions.

While the error detection method is working well, the error correction method in the presented form turned out to be disappointing. This behavior may be explained by the facts, that (1) the accuracy of current electromagnetic tracking systems has still a lot of room for improvement, (2) during system calibration errors are accumulated, and (3), most importantly, field distortions at the flexible sensor differ a lot from those at the rigid sensor in terms of magnitude and direction, although both sensors are quite close to each other. To further improve the error correction and superimposition accuracy, the possible transducer tip movements can be modeled relatively to the rigid body and also the axis of the ultrasound tip can be segmented in the laparoscope images and backprojected into $3 \mathrm{D}$ to further correct the flexible sensor measurements. This is part of our current work and already gave very promising results by reducing dynamic errors of several centimeters to only around $5 \mathrm{~mm}$, contrary to the here described simple correction approach, for which the corrected error grows proportionally to the original error. Additionally, a comparison to and integration of standard EMT calibration techniques is on its way. Our setup could even be used to generate a field distortion function online using the redundancy of the rigid sensor.

\section{Conclusion}

We presented a new method to detect EMT field distortions online by a magnetooptic tracking setup. We improve the state of art [23] for augmenting laparoscopic ultrasound images directly on the laparoscopic live images to give surgeons a better understanding of the spatial relationship between ultrasound and camera images. The laparoscopic ultrasound transducer tip is flexible. Therefore, our method could be applied to a larger set of applications. We are using two attached sensors and hence are able to additionally provide a distrust level of the current EMT measurements. Therefore, the system is able to automatically update and warn the surgical staff of possible inaccuracies. 


\section{References}

1. Harms, J., et al.: Three-dimensional navigated laparoscopic ultrasonography. Surgical Endoscopy 15, 1459-1462 (2001)

2. Leven, J., et al.: Davinci canvas: A telerobotic surgical system with integrated, robot-assisted, laparoscopic ultrasound capability. In: Duncan, J.S., Gerig, G. (eds.) MICCAI 2005. LNCS, vol. 3749, Springer, Heidelberg (2005)

3. Nakamoto, M., et al.: 3d ultrasound system using a magneto-optic hybrid tracker for augmented reality visualization in laparoscopic liver surgery. In: Dohi, T., Kikinis, R. (eds.) MICCAI 2002. LNCS, vol. 2489, Springer, Heidelberg (2002)

4. Ellsmere, J., et al.: A new visualization technique for laparoscopic ultrasonography. Surgery 136, 84-92 (2004)

5. Krücker, J., et al.: An electro-magnetically tracked laparoscopic ultrasound for multi-modality minimally invasive surgery. In: CARS (2005)

6. Kleemann, M., et al.: Laparoscopic ultrasound navigation in liver surgery: technical aspects and accuracy. Surgical Endoscopy 20, 726-729 (2006)

7. Voros, S., Long, J.A., Cinquin, P.: Automatic localization of laparoscopic instruments for the visual servoing of an endoscopic camera holder. In: Larsen, R., Nielsen, M., Sporring, J. (eds.) MICCAI 2006. LNCS, vol. 4190, Springer, Heidelberg (2006)

8. Hummel, J.B., et al.: Design and application of an assessment protocol for electromagnetic tracking systems. Medical Physics 32, 2371-2379 (2005)

9. Nafis, C., Jensen, V., Beauregard, L., Anderson, P.: Method for estimating dynamic em tracking accuracy of surgical navigation tools. In: Medical Imaging 2006: Visualization, Image-Guided Procedures, and Display (2006)

10. Kindratenko, V.V.: A survey of electromagnetic position tracker calibration techniques. Virtual Reality: Research, Development, and Applications 5, 169-182 (2000)

11. Birkfellner, W., et al.: Concepts and results in the development of a hybrid tracking system for cas. In: Wells, W.M., Colchester, A.C.F., Delp, S.L. (eds.) MICCAI 1998. LNCS, vol. 1496, Springer, Heidelberg (1998)

12. Sielhorst, T., Feuerstein, M., Traub, J., Kutter, O., Navab, N.: Campar: A software framework guaranteeing quality for medical augmented reality. International Journal of Computer Assisted Radiology and Surgery 1, 29-30 (2006)

13. Daniilidis, K.: Hand-eye calibration using dual quaternions. International Journal of Robotics Research 18, 286-298 (1999)

14. Yamaguchi, T., et al.: Development of a camera model and calibration procedure for oblique-viewing endoscopes. Computer Aided Surgery 9, 203-214 (2004)

15. Treece, G.M., et al.: High-definition freehand 3-d ultrasound. Ultrasound in Medicine and Biology 29, 529-546 (2003)

16. Lang $\varnothing$, T.: Ultrasound Guided Surgery: Image Processing and Navigation. PhD thesis, Norwegian University of Science and Technology (2000) 